\title{
Capacidad predictiva de la rentabilidad en empresas del mercado de capitales de Argentina
}

\author{
Predictive capacity of profitability in companies \\ in the capital market of Argentina
Dante Domingo Terreno *, Silvana Andrea Sattler y Enrique Leopoldo Castro González

Universidad Nacional Córdoba, Argentina

Recibido el 05 de julio de 2016; aceptado el 05 de diciembre de 2017

Disponible en Internet el 27 de septiembre de 2018

\section{Resumen}

Farfield y Yohn (2001) presentan un sencillo e interesante modelo a efectos de predecir la rentabilidad en el corto plazo, en base a la rentabilidad actual y los componentes de Dupont. No obstante, la importancia y difusión de dicho modelo no ha sido estudiado en economías emergentes. Es por ello, que en el presente trabajo se plantea como objetivo evaluar la capacidad predictiva de la rentabilidad de los activos operativos netos, en base al modelo mencionado en el mercado de capitales de Argentina. Los modelos contrastados muestran un importante proceso de reversión a la media de rentabilidad y que solamente la rotación de los activos operativos netos permite la mejoría en la rentabilidad del año siguiente. En cambio, el aumento del margen de ganancias revierte rápidamente, determinando un efecto negativo sobre rentabilidad del año siguiente.

Códigos JEL: M21, M41, G17.

Palabras claves: Reversión a la media; Contabilidad financiera; Análisis de estados financieros.

\footnotetext{
*Autor para correspondencia.

Correo electrónico: danteterreno@ fibertel.com.ar (D. Domingo Terreno)

La revisión por pares es responsabilidad de la Universidad Nacional Autónoma de México.
} 


\begin{abstract}
Farfield and Yohn (2001) develop a simple and interesting model to predict the effects of profitability in the short term, based on current profitability and the components of Dupont. However, the importance and dissemination of this model has not been estudied in emerging economies. For this reason, document has as purpose to evaluate the predictive ability of the return on net operating assets, based on the above model in the Argentina capital market. The models checked show a strong process of mean reversion of returns, and only the rotation of the net operating assets allows the improvement in profitability in the next year. Instead, the profit margin increased rapidly reversed, determining a negative effect on profitability in the next year.
\end{abstract}

JEL classification: M21, M41, G17.

Keywords: Mean reversion; Financial accounting; Financial statement analysis.

\title{
Introducción
}

En uno de los trabajos pioneros del análisis fundamental, Lev y Thiagarajan (1993) señalan que la mayor utilidad de la información contable es permitir a los inversores pronosticar la persistencia y crecimiento de los resultados futuros. Las economías desarrolladas, en especial en Estados Unidos, han desarrollado una amplia investigación sobre el pronóstico de la rentabilidad. En países emergentes no ha ocurrido lo mismo, las investigaciones en contabilidad financiera se han focalizado en diversos temas, tales como los modelos de predicción de insolvencia, el retorno de las acciones y calidad de la información contable (v.g. Sandin y Porporato, 2007; Swanson, Rees y Juarez-Valdes, 2003; Mahmud, Ibrahim y Pok, 2009). Pero, con respecto a los modelos de predicción de la rentabilidad futura la bibliografía muestra que no ha existido un mayor interés. En el mismo sentido, la descomposición de la rentabilidad por Dupont en margen de ganancias y rotación de los activos, mencionado en los libros de texto, es aplicado para explicar la rentabilidad actual y no para la predicción de la rentabilidad.

La rotación de los activos mide la habilidad de la empresa para generar ingresos por la utilización de los activos, mientras que el margen de ganancias mide la habilidad de controlar los costos en relación con los ingresos (Penman y Zhang, 2002). Estas son determinadas por las estrategias de la empresa, la diferenciación está basada en el margen de ganancia y el liderazgo en costos en la rotación (Selling y Stickney 1989). Las evidencias señalan que la desagregación de la rentabilidad actual por los componentes de Dupont incrementa la capacidad predictiva de la rentabilidad (v.g. Soliman, 2004; Farfield y Yohn, 2001).

Uno de los modelos más difundidos en la predicción de la rentabilidad es el desarrollado por Farfield y Yohn (2001), donde la rentabilidad es representada por la rentabilidad de los activos operativos netos (RNOA). Dicho estudio permite testear la teoría de reversión a la media de la rentabilidad (Stigler, 1963; Muller, 1977; Fama y French, 2000), el impacto de las nuevas inversiones y el efecto del cambio del margen de ganancias y rotación de los activos, sobre el cambio de la RNOA del año siguiente. La importancia del estudio de la rentabilidad operativa se justifica en el hecho que las actividades operativas son las que generan los resultados anormales (Ohlson, 1995). No obstante, la gran difusión del modelo mencionado, no han sido aplicado en la predicción de la rentabilidad en economías emergentes. También, la desagregación de Dupont ha sido aplicada en los modelos de predicción de la insolvencia y rendimiento de las acciones (v.g. Sandin y Porporato 2007; Zanjirdar, Khaleghi Kasbi y Madahi, 2014). 
La Argentina es un caso de una economía emergente e inestable bastante ejemplificativo. Posterior a una fuerte recesión (1999-2002) se inicia un período de rápida recuperación, pero a partir del año 2007 comienzan a aparecer problemas inflacionarios a medida que se va cubriendo la capacidad ociosa, lo que lleva a partir del año 2012 a un proceso de estancamiento con inflación (Anuarios IAMC, 2003 a 2012). Entonces, dada la sustancial diferencia entre el contexto de una economía emergente y una avanzada, es académicamente interesante analizar si los modelos desarrollados en un contexto determinado aplicarlos en otro. Así, en el presente trabajo, se plantea como objetivo evaluar la capacidad predictiva de RNOA actual y el margen de ganancias y la rotación de activos en el mercado de capitales de Argentina, tomando como base el modelo de Farfield y Yohn (2001). Adicionalmente, es avaluada la capacidad predictiva de la combinación conjunta de los cambios de los componentes de Dupont (Penman y Zhang, 2002) y el pronóstico individual de los mismos.

Konchitchki (2011) señala que la distorsión en los estados financieros en moneda nominal tiene implicancias en la evaluación del rendimiento y precio de las acciones, incluso cuando la inflación es baja. En Argentina, a pesar de tener una inflación que supera dos dígitos los estados financieros no son ajustados por inflación. Por lo tanto, se aplicará un procedimiento de ajuste por inflación a los estados publicados a efectos de disminuir el sesgo en la información contable.

Este trabajo intenta ser un aporte a los investigadores y profesionales en el pronóstico de la rentabilidad futura, para la determinación del valor de las acciones y la capacidad de pago de las obligaciones de la empresa.

\section{Marco teórico}

Desde el punto de vista de inversor, el objetivo de la investigación empírica de archivo es entender las propiedades de la información financiera y cómo esta información podría ayudar generar mejores pronósticos (Richardson, Tuna y Wysocki, 2010). La investigación ha elaborado distintos modelos para la predicción de la rentabilidad: (1) en base a la rentabilidad actual o de ejercicios precedentes (v.g. Lipe y Kormendi, 1994; Canarella, Millery y Nourayi, 2013); (2) por la desagregación de los componentes de la rentabilidad (v.g. Fairfield, Sweene y Yohn, 1996); (3) por el pronóstico de los componentes de la rentabilidad (Monterrey Mayoral y Sánchez Segura, 2016); (4) por la rentabilidad actual, y el efecto adicional de la desagregación de sus componentes (v.g. Farfield y Yohn, 2001; Soliman, 2004).

Una de las principales teorías que explica la relación entre la rentabilidad actual y futura de una empresa es la reversión a la media de la rentabilidad. La idea básica es que en una economía competitiva los beneficios por encima o por debajo de la media deberían desaparecer revirtiendo a la media. Uno de los pioneros de esta teoría, Stigler (1963) afirma que las diferencias en las tasas de rentabilidad en las diferentes industrias no se pueden mantener a lo largo del tiempo. Por otra parte, Mueller (1977) analiza la convergencia de la rentabilidad, entendida como el resultado antes de impuestos dividido por el total de activos. El estudio rechaza la hipótesis de un ambiente competitivo y de una rápida convergencia, en cambio señala que la reversión a la media depende de la estructura del mercado, por ejemplo, la existencia de concentración y barreras de entrada. Fama y French (2000) sostienen que la reversión a la media es más rápida cuando la rentabilidad está por debajo de su media o cuando está más lejos de su media. El estudio de Lipe y Kormendi (1994), también, proveen una fuerte evidencia de la propiedad 
de reversión a la media, medido por el resultado por acción con un modelo ARIMA $^{2}$ de alto orden. Los estudios basados en el modelo de Farfield y Yohn (2001) confirman la reversión a la media de la RNOA (Farfield y Yohn 2001; Soliman 2004; Penman y Zhang 2002; Monterrey y Sánchez-Segura 2011).

El aumento de las inversiones, por lo general, es un buen augurio de crecimiento de los resultados futuros y de flujos futuros de efectivo (Lev y Thiagarajan, 1993). La evidencia de dicho estudio, indica la existencia de una relación positiva entre el crecimiento de la inversión de capital de la empresa con los resultados futuros, medido en término relativos con el crecimiento promedio de la industria. Al contrario, otros estudios sostienen que los aumentos en las inversiones de capital tienen una relación inversa con los subsecuentes cambios en los resultados futuros (Ou, 1990; Arbanel y Bushee, 1997). Los nuevos proyectos de capital generalmente no afectan los resultados futuros en forma inmediata por el cargo de la depreciación (Arbanel y Bushee, 1997). Missim y Penman (2001) encuentran una relación positiva entre el incremento de los activos operativos netos y la rentabilidad de los activos operativos netos actual, aunque, señalan que podría ser negativa si se aplica una política contable conservadora. Una política de depreciación y amortización acelerada de las nuevas inversiones produce una disminución de la rentabilidad en los primeros años. Otros trabajos, documentan una relación negativa en el crecimiento de los activos operativos netos y la rentabilidad futura de los activos operativos netos (Farfield y Yohn, 2001; Soliman, 2004; Penman y Zhang, 2002).

El estudio de Farfield y Yohn (2001) que incluye los resultados negativos, concluye en que la descomposición entre el margen y rotación mejora la predicción de la rentabilidad del año siguiente, no por la combinación de ambos sino por el cambio en dichos componentes. La evidencia muestra que el cambio en la rotación de activos es más perdurable que el cambio en el margen de ganancia, este último, tiene un efecto negativo o no significativo (Farfield y Yohn, 2001; Penman y Zhang, 2002; Monterrey y Sánchez-Segura, 2011).

Referidos a la calidad de la información contable, Penman y Zhang (2002) sostienen que cuando el cambio del margen y rotación se mueven en sentido contrario puede indicar un problema con la calidad de la información contable. Jansen, Ramnath y Yohn (2011) sostienen que cuando PM (margen de ganancias) y ATO (rotación de activos) se mueven en dirección opuesta los resultados están siendo afectados por earnings management. La evidencia muestra que el diagnóstico de ATO-PM es útil para predecir el decrecimiento (incremento) en el año siguiente asociado con la reversión de aumento (disminución) en el período actual. Un estudio similar para empresas de la bolsa de valores de Teherán, el trabajo de Hejazi, Adampira y Bahrami (2016) llegan a las mismas conclusiones de estudio anterior, pero no precisa como se arriban a las mismas.

Por otra parte, Amir, Kama y Live (2015) examinan la reacción del mercado a los coeficientes de Dupont en base a estados contables trimestrales y encuentran que la persistencia incondicional de la ATO es mayor que la del PM, pero la persistencia condicional de PM es mayor que la del ATO y tiene un efecto positivo. La persistencia condicional se define como la contribución marginal de la persistencia de una variable a la persistencia de un variable de más alta en la jerarquía. Zanjirdar y otros (2014) para empresas de la bolsa de valores de Teherán, encuentran que en el comportamiento de los inversores tiene un mayor efecto el margen de ganancias que la rotación, efecto solo sostenible en el corto plazo.

\footnotetext{
2 ARIMA: Modelo Autorregresivo Integrado de Medias Móviles.
} 
En síntesis, de la revisión bibliográfica surge que solo el incremento en la rotación de los activos produce un aumento de la rentabilidad de los activos operativos netos sostenible, mientras que, el incremento del margen de ganancias tiene un efecto negativo o no significativo. Por otra parte, cuando el margen de ganancias y la rotación de activos se mueven en sentido contrario y al año siguiente se produce la reversión de dichos cambios, es considerado el efecto de earnings management. Por último, en el pronóstico del rendimiento de las acciones el margen de ganancias muestra un efecto positivo.

\section{Presentación del contexto de aplicación}

\section{Economías emergentes}

En los últimos veinte años, las economías emergentes han crecido en mayor medida que las economías desarrolladas, impulsados por los países del BRIC (Brasil, Rusia, India y China). Las economías emergentes han aumentado su participación en el producto global del $30 \%$ en 1990 a más del 50\%, de acuerdo con datos del Fondo Monetario Internacional (The economist, 2013). No obstante, las economías emergentes están expuestas a fuertes desequilibrios. Los ciclos económicos son más severos que en las economías desarrolladas, se caracterizan, cada vez más, por su gran volatilidad y dramáticas reversiones de la cuenta corriente, fenómeno denominado de "parada repentina". Por otra parte, existe una mayor variabilidad en el consumo que en los ingresos, la tasa de interés suele ser contra cíclica y prevalen las tendencias a los shocks económicos (Notz y Rosenkranz 2014; Aguiar y Gopinath 2007).

\section{Economía Argentina}

La Argentina es un ejemplo paradigmático de una economía emergente. En el año 2002 se produce una fuerte crisis en la economía argentina, tras la decisión de no pago de la deuda externa, se produjo un cambio de las reglas de juego de la economía. La fuerte devaluación del tipo de cambio benefició a los sectores agropecuario, industrial y minero, el congelamiento de las tarifas hizo caer la rentabilidad de las empresas de sector servicios públicos.

Tabla 1

Indicadores Económicos

\begin{tabular}{ccc}
\hline Año & $\begin{array}{c}\text { Variación PBI a } \\
\text { precios } 1993(1)\end{array}$ & $\begin{array}{c}\text { Variación } \\
\text { anual IPC (2) }\end{array}$ \\
\hline 2012 & 1.90 & 23.01 \\
2011 & 8.87 & 23.28 \\
2010 & 9.16 & 27.03 \\
2009 & 0.85 & 18.47 \\
2008 & 6.76 & 20.60 \\
2007 & 8.65 & 21.52 \\
2006 & 8.47 & 9.40 \\
2005 & 9.18 & 12.33 \\
2004 & 9.03 & 6.10 \\
2003 & 8.84 & 3.66 \\
2002 & -10.98 & 40.95 \\
\hline
\end{tabular}

Fuente: Elaboración propia en base datos:

(1) INDEC y (2) del 2002-2006 INDEC y 2007-2012 DPEyC San Luis. 
El congelamiento de los salarios llevó a una mejora de la rentabilidad de las empresas. Adicionalmente, las empresas endeudadas en dólares fueron beneficiadas con un tipo de cambio diferencial. En el estado, las retenciones a las exportaciones, el no pago de intereses de la deuda y los salarios congelados produjeron la obtención de un superávit fiscal. La caída del PBI fue de casi el $11 \%$ y una inflación medida por precios al consumidor del $40.95 \%$, ver tabla 1.

A partir de año 2003, la economía vuelve a crecer de manera importante observándose una recuperación del consumo, inversión y exportaciones, motivada por la suba del precio internacional de los commodities y la capacidad instalada ociosa de la industria. En los años posteriores continuó el crecimiento a elevadas tasas, salvo en el año 2009 por la crisis mundial desencadenada a mediados del 2008. Pero, a partir del año 2012, la economía entra en un proceso de desaceleración económica (Anuarios IAMC, 2003 a 2012). En otra cuestión, a partir del año 2007 se produce una aceleración del ritmo inflacionario, situación que no es reflejada por las estadísticas oficiales. Un informe Fondo Monetario Internacional (2013) hace referencia a la declaración de censura por parte del FMI para Argentina y donde se le solicita medidas correctivas para mejorar la calidad de los datos oficiales del PBI y del índice de precios del consumidor. Para sustituir las estadísticas oficiales es utilizada una fuente más confiable como los índices de precios publicados por Dirección de Estadística de la Provincia de San Luis $^{3}$.

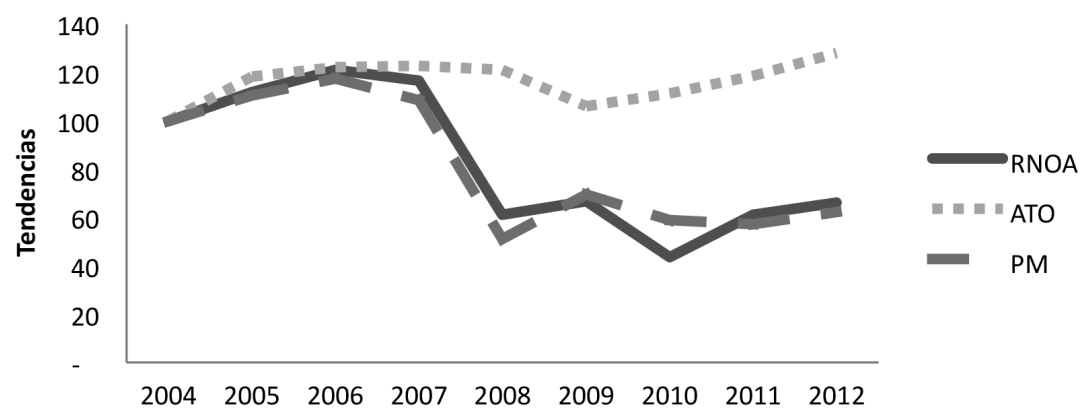

Figura 1. Tendencias de las principales ratios contables Fuente: Elaboración propia.

La figura 1 presenta las tendencias de la media anual de RNOA, ATO y PM en el período de estudio. Las tendencias fueron calculadas en porcentajes, con base en el año 2004. En la mayor parte de los años el margen y rotación varían en sentido contrario. Salvo, en los dos primeros años y el último, donde varían en igual dirección, probablemente por la existencia de capacidad ociosa originada en la caída de la actividad de años anteriores. Analizados de manera individual, el margen experimenta un brusco descenso a partir del año 2007, posiblemente, afectado por la aceleración de la inflación que modifica los precios relativos y por los propios problemas de la economía argentina. La rotación muestra una mayor estabilidad que el margen de ganancias, si bien refleja el impacto de la crisis internacional del año 2009. La explicación de la evolución de la RNOA se explica por la evolución del margen y rotación.

\footnotetext{
${ }^{3}$ Este mismo índice es utilizado por IAMC para el ajuste de sus datos.
} 


\section{Metodología de la investigación}

\section{Datos}

El objeto del estudio es el conjunto de empresas autorizadas a cotizar sus acciones en la Bolsa de Comercio de Buenos Aires en el período 2005-2012, en base a los estados contables anuales consolidados de dichas empresas. A la muestra inicial se la depuró eliminando aquellas observaciones (empresas-año) que cumplan algunas de las siguientes condiciones: (1) las empresas de actividad financiera; (2) las empresas cuyos estados contables consolidados muestren cifras de patrimonio neto, ventas anuales y activos operativos netos menores a $\$ 1000000$; (3) los estados contables ${ }^{4}$ que no se encuentren confeccionados de acuerdo a las Normas Contables Profesionales Argentinas (NCPs), por una cuestión de consistencia; (4) empresas de capitales extranjeros.

La población de empresas, ya depuradas, está constituida por un total 80 empresas a las que corresponde un total de 500 observaciones. El tratamiento de los datos atípicos (outliers) consistió en eliminar el $6 \%$ de las observaciones con residuos de mayor valor en términos absolutos, dicho porcentaje surge del análisis de sensibilidad de las observaciones consideradas influyentes. La muestra final, después que se eliminaron los outliers es de 470 observaciones.

\section{Variables y estadísticos descriptivos}

Esplin, Hewitt, Plumlee y Yohn (2013), para la predicción de la rentabilidad del patrimonio neto (ROI), sostienen que la desagregación en componentes financieros y operativos tiene un muy pequeño efecto, mientras que, la RNOA antes de partidas infrecuentes e inusuales supera claramente el modelo agregado. En base a dicha evidencia, en este estudio la rentabilidad es analizada por las partidas permanentes de los resultados.

Para el cálculo de la RNOA se siguió el apéndice de Missim y Penman (2001), siendo:

a) RNOA: mide la rentabilidad de la empresa excluyendo los resultados de las actividades de financiación y las partidas anormales, y se expresa como:

$$
\text { RNOA }=\frac{\text { Resultados operativos }(O I)}{\text { Activos operativos netos (NOA) promedio }}
$$

El promedio surge de la suma del saldo inicial y el saldo final, dividido por 2.

Donde el numerador es igual a:

$$
O I=R E-R E x t r-R D i s c o n t-O t r o s I E+N F E[1]
$$

Siendo que:

RE: Resultadodel ejercicio;

RExtr: Resultados extraordinarios;

RDiscont: Resultados de operaciones en discontinuación;

OtrosIE: Otros ingresos y egresos;

NFE: Gastos financieros netos.

\footnotetext{
${ }^{4}$ Estados Contables es la denominación en Argentina de los estados financieros.
} 
Donde los gastos financieros netos (NFE), de la expresión [1], son determinados por:

$$
N F E=(G F-I F) x(1-T I)
$$

Siendo que:

$G F$ : Gastos financieros;

$I F$ : Ingresos financieros;

TI: Tasa impositiva.

Donde el denominador es igual a:

$$
N O A=O A-O L
$$

Donde los activos operativos (OA) son iguales a:

$$
O A=T A-F A[3]
$$

Siendo que:

OA: Activos operativos

TA: Total de activos

FA: Activos financieros

Donde los activos financieros (FA), de la expresión [3], es igual a:

$$
F A=\text { Caja y bancos }+ \text { Inversiones transitorias }+ \text { Otras inversiones }
$$

Donde los pasivos operativos (OL), de la expresión [2], es igual a:

$$
O L=\text { Pasivos totales }(T L)-\text { Préstamos a corto y largo plazo }
$$

Siendo que:

TL: Pasivos totales

b) Margen de ganancias (PM): componente de la desagregación de Dupont y se obtiene por la siguiente expresión:

$$
P M=\frac{I O}{\text { Ventas }}
$$

c) Rotación de los activos operativos netos (ATO): componente de la desagregación de Dupont y se obtiene por la siguiente expresión:

$$
A T O=\frac{\text { Ventas }}{\text { NOA promedio }}
$$

d) Los cambios en las variables surgen de las expresiones siguientes:

$$
\Delta R N O A_{t+1}=R N O A_{t+1}-R N O A_{t}
$$


Siendo:

$\Delta \mathrm{RNOA}_{\mathrm{t}+1}$ : Cambio en rentabilidad de los activos operativos netos del año siguiente;

RNOA $_{t}$ : Rentabilidad de los activos operativos netos del año actual.

$$
\operatorname{CrNOA}=\frac{\operatorname{CrNOA}_{t}}{\operatorname{CrNOA_{t-1}}}-1
$$

Siendo:

$\mathrm{CrNOA}_{\mathrm{t}}$ : Incremento en los activos operativos netos del año actual.

Siendo:

$$
\begin{aligned}
& \Delta A T O_{t}=A T O_{t}-A T O_{t-1} \\
& \Delta P M_{t}=P M_{t}-P M_{t-1}
\end{aligned}
$$

$\triangle A T O_{t}$ : Cambio en la rotación de los activos operativos netos del año actual;

$\Delta P M_{t}$ : Cambio en el margen de ganancia de año actual.

\section{Ajuste por inflación de los estados contables}

La Argentina enfrenta desde el año 2005 una inflación superior a los dos dígitos y de acuerdo con la normativa contable vigente son emitidos en moneda nominal, por lo que se encuentran seriamente sesgados. Debido a dicha distorsión, resulta necesaria la aplicación de un procedimiento de ajuste a efectos de atenuar el efecto de la misma. El algoritmo de ajuste utilizado sigue en líneas generales el procedimiento establecido por la Resolución Técnica $\mathrm{N}^{\circ} 6$ (RT 6) de las $\mathrm{NCPs}^{5}$, aunque, es aplicado a los activos, pasivos y resultados operativos. Dicho algoritmo de ajuste tiene dos pasos:

a) Por un lado, se ajusta el saldo inicial y la variación en el ejercicio de las partidas no monetarias de los activos operativos netos valuadas al costo (bienes de uso, intangibles y llave de negocio); y por otro, el activo operativo neto sin incluir el resultado operativo del ejercicio.

b) Basado en la identidad contable, el resultado operativo del ejercicio ajustado surge por la diferencia entre el activo operativo ajustado, el pasivo operativo ajustado y el activo operativo neto ajustado sin incluir el resultado operativo del ejercicio.

Los saldos al inicio del ejercicio son ajustados por el coeficiente del inicio al cierre del ejercicio y las variaciones del ejercicio por el coeficiente del promedio del ejercicio al cierre. El procedimiento de ajuste se efectúa en forma acumulativa, a partir del momento en que se discontinuó del ajuste por inflación en marzo 2003. Para el cálculo de los coeficientes de ajuste, para el período 2002-2006 se utiliza el Índice de Precios al Consumidor elaborado por el Instituto Nacional de Estadísticas y Censos de la República Argentina (INDEC). A partir del año 2007, debido a la falta de confiabilidad de los índices publicados por el INDEC, se recure a los índices de Precios al Consumidor elaborado Dirección de Estadística y Censos de la Provincia de San Luis (DPEyC-San Luis) ${ }^{6}$.

${ }^{5}$ NCPs: normas contables emitidas por la Federación Argentina de Consejo Profesional de Ciencias Económicas (FACPCE).

${ }^{6}$ Este mismo índice es utilizado por Instituto Argentino de Mercado de Capitales (IAMC) para el ajuste de sus datos. 


\section{Modelos}

Los modelos que se contrastan en este trabajo se corresponden con el grupo de modelos mencionado como (4) de la clasificación efectuada en el marco teórico, donde la rentabilidad del año siguiente es pronosticada por la rentabilidad actual y la desagregación de sus componentes. Los cuáles serán comparados con el modelo donde la rentabilidad futura en pronosticada solamente en base a la rentabilidad actual. Los mismos son expuestos a continuación:

\section{Modelo I}

El modelo reversión se plantea como:

$$
\Delta R N O A_{t+1}=\propto+\beta_{1} R N O A_{t}+\varepsilon_{t+1}
$$

Siendo:

$\varepsilon_{t+1}$ : Término de error aleatorio del período $t+1$.

Este modelo permite comprobar el grado de reversión a la media de la RNOA actual con respecto al RNOA del año próximo, el signo esperado es negativo.

\section{Modelo II}

Incorpora al primer modelo, a través de variables “dicotómicas", el efecto de la interacción de los signos (aumento o disminución) del $\Delta \mathrm{ATO}_{\mathrm{t}}$ y $\Delta \mathrm{PM}_{\mathrm{t}}$, de acuerdo al modelo utilizado por Penman y Zhang (2002). La expresión matemática es:

$$
\triangle R N O A_{t+1}=\propto+\beta_{1} R N O A_{t}+\beta_{2} R M 1_{t}+\beta_{3} R M 2_{t}+\beta_{4} R M 3_{t}+\varepsilon_{t+1}
$$

Siendo:

RM1: es igual a 1 sí $\left(\Delta \mathrm{ATO}_{\mathrm{t}}<0\right.$ y $\left.\Delta \mathrm{PM}_{\mathrm{t}}>0\right)$;

RM2: es igual a 1 sí $\left(\Delta \mathrm{ATO}_{\mathrm{t}}>0\right.$ y $\left.\Delta \mathrm{PM}_{\mathrm{t}}<0\right)$;

RM3: es igual a 1 sí $\left(\Delta \mathrm{ATO}_{\mathrm{t}}<0\right.$ y $\left.\Delta \mathrm{PM}_{\mathrm{t}}<0\right)$

La ordenada de origen $(\propto)$ captura el efecto de $\left(\Delta \mathrm{ATO}_{\mathrm{t}}>0\right.$ y $\left.\Delta \mathrm{PM}_{\mathrm{t}}>0\right)$

Cuando, ambos, $\Delta \mathrm{PM}_{\mathrm{t}}$ y $\Delta \mathrm{ATO}_{\mathrm{t}}$ son positivos (aumento) se espera el mayor impacto sobre el esta situación es muy posible cuando existen costos fijos importantes y el mayor nivel de ventas reduce la incidencia de estos. En caso de signos contrarios, permite diagnosticar el efecto de earnings managment, reflejado por los coeficientes de RM1 negativo y RM2 positivo.

\section{Modelo III}

Este modelo incorpora al anterior el crecimiento de los activos operativos netos ( $\mathrm{CrNOA}_{\mathrm{t}}$ ), el cambio en la rotación $\left(\Delta \mathrm{ATO}_{\mathrm{t}}\right)$ y el cambio en el margen $\left(\Delta \mathrm{PM}_{\mathrm{t}}\right)$, a efecto de predecir el cambio de la RNOA del año posterior. La expresión matemática es:

$$
\Delta R N O A_{t+1}=\propto+\beta_{1} R N O A_{t}+\beta_{2} \operatorname{CrNOA}_{t}+\beta_{3} \Delta A T O_{t}+\beta_{4} \Delta P M_{t}+\varepsilon_{t+1}
$$


El modelo intenta determinar la capacidad predictiva del $\Delta \mathrm{ATO}_{\mathrm{t}}$ y del $\Delta \mathrm{PM}_{\mathrm{t}}$, al cual se agrega el $\mathrm{CrNOA}_{t}$ a efecto de controlar el efecto de las nuevas inversiones. De acuerdo a los estudios se espera un coeficiente del $\mathrm{CrNOA}_{\mathrm{t}}$ negativo, del $\Delta \mathrm{ATO}_{\mathrm{t}}$ positivo y del $\Delta \mathrm{PM}_{\mathrm{t}}$ negativo o no significativo (Farfield y Yohn, 2001; Penman y Zhang, 2002; Bauman, 2014).

\section{Modelo IV}

En este caso se incorpora al tercer modelo, a través de variables "dicotómicas", la interacción de los signos positivos y negativos (aumento o disminución) del $\Delta \mathrm{ATO}_{\mathrm{t}}$ y $\Delta \mathrm{PM}_{\mathrm{t}}$. La expresión matemática es:

$$
\begin{aligned}
\Delta \mathrm{RNOA}_{\mathrm{t}+1}=\propto & +\beta_{1} \mathrm{RNOA}_{\mathrm{t}}+\beta_{2} \mathrm{CrNOA}_{\mathrm{t}}+\beta_{3} \Delta \mathrm{ATO}_{\mathrm{t}}+\beta_{4} \Delta \mathrm{PM}_{\mathrm{t}}+\beta_{5} R M 1_{t}+\beta_{6} R M 2_{t} \\
& +\beta_{7} R M 3_{t}+\varepsilon_{\mathrm{t}+1}
\end{aligned}
$$

Este modelo, además, de analizar las variables del modelo anterior controla el posible efecto de, mencionado en el modelo II.

\section{Modelo V}

Este modelo, el $\Delta \mathrm{RNOA}_{\mathrm{t}+1}$ está en función de la $\mathrm{RNOA}_{\mathrm{t}}$, el $\Delta \mathrm{PM}_{\mathrm{t}} \mathrm{y}$ las variables "dicotómicas" de la interacción de los signos positivos y negativos (aumento o disminución) del $\mathrm{CrNOA}_{\mathrm{t}}$ y $\Delta \mathrm{ATO}_{\mathrm{t}}$, de acuerdo con el modelo utilizado por Penman y Zhang (2002). La expresión matemática es la siguiente:

$$
\Delta R N O A_{t+1}=\propto+\beta_{1} R N O A_{t}+\beta_{2} \Delta P M_{t}+\beta_{3} G R_{t}+\beta_{4} G R 2_{t}+\beta_{5} G R 3_{t}+\varepsilon_{t+1}
$$

Siendo

GR1: es igual a 1 sí $\left(\Delta \mathrm{ATO}_{\mathrm{t}}>0\right.$ y $\left.\mathrm{CrNOA}_{\mathrm{t}}<0\right)$;

GR2: es igual a 1 sí $\left(\Delta \mathrm{ATO}_{\mathrm{t}}<0\right.$ y $\left.\mathrm{CrNOA}_{\mathrm{t}}<0\right)$;

GR3: es igual a 1 sí $\left(\Delta \mathrm{ATO}_{\mathrm{t}}<0\right.$ y $\left.\mathrm{CrNOA}_{\mathrm{t}}<0\right)$

La ordenada de origen $(\alpha)$ captura el efecto de $\left(\Delta \mathrm{ATO}_{\mathrm{t}}>0\right.$ y $\left.\mathrm{CrNOA}_{\mathrm{t}}>0\right)$.

Este modelo trata de evaluar el efecto adicional $\Delta \mathrm{PM}_{\mathrm{t}}$ y $\Delta \mathrm{ATO}_{\mathrm{t}}$, pero esta última a través de la interacción entre el $\mathrm{CrNOA}_{\mathrm{t}}$ y $\Delta \mathrm{ATO}_{\mathrm{t}}$. Se espera que el mayor efecto sobre el $\Delta \mathrm{RNOA}_{\mathrm{t}+1}$ sea producido por $\left(\Delta \mathrm{ATO}_{\mathrm{t}}>0\right.$ y $\left.\mathrm{CrNOA}_{\mathrm{t}}>0\right)$, situación donde las nuevas inversiones producen un mayor nivel de ventas. Cuando el $\mathrm{CrNOA}_{t}$ y $_{\Delta \mathrm{ATO}_{\mathrm{t}}}$ se mueven en sentido contrario se espera un efecto positivo GR1, debido a que $\Delta \mathrm{RNOA}_{\mathrm{t}+1}$ aumenta cuando el $\Delta \mathrm{ATO}_{\mathrm{t}}$ es positivo (Penman y Zhang, 2002).

\section{Modelo VI}

En este caso, se incorpora al tercer modelo la interacción entre los signos de $\mathrm{CrNOA}_{t}$ y el $\Delta \mathrm{ATO}_{\mathrm{t}}$. La expresión matemática es:

$$
\begin{aligned}
\Delta \mathrm{RNOA}_{\mathrm{t}+1}= & \propto \\
& +\beta_{1} \mathrm{RNOA}_{\mathrm{t}}+\beta_{2} \mathrm{CrNOA}_{\mathrm{t}}+\beta_{3} \Delta \mathrm{ATO}_{\mathrm{t}}+\beta_{4} \Delta \mathrm{PM}_{\mathrm{t}}+\beta_{5} G R 1_{t}+\beta_{6} G R 2_{t} \\
& +\beta_{7} G R 3_{t}+\varepsilon_{\mathrm{t}+1}
\end{aligned}
$$


Además, en este estudio para profundizar el análisis de los componentes de Dupont se efectúa el pronóstico individual de los mismos para el año siguiente, por lo cual se plantean los siguientes modelos:

\section{Modelo a}

$$
\Delta A T O_{t+1}=\propto+\beta_{1} A T O+\beta_{2} \Delta A T O_{t}+\varepsilon_{t+1}
$$

\section{Modelo b}

$$
\Delta P M_{t+1}=\propto+\beta_{1} P M+\beta_{2} \Delta P M_{t}+\varepsilon_{t+1}
$$

En la tabla 2 se presentan los estadísticos descriptivos de cada una de las variables. De los datos se destaca una $\operatorname{RNOA}_{t}(4.5736 \%)$ que es muy inferior al de otros estudios en países desarrollados, por ejemplo, Missim y Penman (2001) $\left(\mathrm{RNOA}_{\mathrm{t}}=11.10 \%\right)$ y Soliman (2004) $\left(\mathrm{RNOA}_{\mathrm{t}}=13.07 \%\right)$, lo cual surge de un menor margen y rotación. Aunque, los datos pueden no ser totalmente comparables debido a la aplicación de normas contables distintas.

Tabla 2

Estadísticos descriptivos

\begin{tabular}{ccc}
\hline Variables & Media & Desv.Típ. \\
\hline$\Delta$ RNOA $_{\mathrm{t}+1}$ & -0.8382 & 6.181 \\
RNOA $_{\mathrm{t}}$ & 4.5736 & 9.063 \\
CrNOA $_{\mathrm{t}}$ & 3.9014 & 13.820 \\
$\Delta \mathrm{ATO}_{\mathrm{t}}$ & 0.0148 & 0.269 \\
$\Delta \mathrm{PM}_{\mathrm{t}}$ & -0.9372 & 8.197 \\
$\Delta \mathrm{ATO}_{\mathrm{t}+1}$ & -0.0995 & 0.112 \\
$\Delta \mathrm{PM}_{\mathrm{t}+1}$ & -0.7619 & 0.326 \\
$\mathrm{ATO}_{\mathrm{t}}$ & 1.3278 & 0.454 \\
$\mathrm{PM}_{\mathrm{t}}$ & 4.0590 & 0.513 \\
\hline
\end{tabular}

Fuente: Elaboración propia

En la tabla 3 se presentan las correlaciones de Spearman y se destaca la importante correlación entre el $\mathrm{PM}_{\mathrm{t}}$ con respecto a $\mathrm{RNOA}_{\mathrm{t}}$ y el $\Delta \mathrm{PM}_{\mathrm{t}+1}$ con la $\mathrm{RNOA}_{\mathrm{t}+1}$. 
Tabla 3

Correlación de Spearman entre las variables

\begin{tabular}{llllllllll}
\hline Variables & $\Delta \mathrm{RNOA}_{\mathrm{t}+1}$ & $\mathrm{RNOA}_{\mathrm{t}}$ & $\mathrm{GrNOA}_{\mathrm{t}}$ & $\Delta \mathrm{ATO}_{\mathrm{t}}$ & $\Delta \mathrm{PM}_{\mathrm{t}}$ & $\mathrm{ATO}_{\mathrm{t}}$ & $\mathrm{PM}_{\mathrm{t}}$ & $\Delta \mathrm{ATO}_{\mathrm{t}+1}$ & $\Delta \mathrm{PM}_{\mathrm{t}+1}$ \\
$\Delta \mathrm{RNOA}_{\mathrm{t}+1}$ & 1.0000 & & & & & & & & \\
RNOA $_{\mathrm{t}}$ & -0.4017 & 1.0000 & & & & & & & \\
CrNOA $_{\mathrm{t}}$ & -0.2437 & 0.2529 & 1.0000 & & & & & & \\
$\Delta \mathrm{ATO}_{\mathrm{t}}$ & & 0.1203 & -0.2240 & 1.0000 & & & & & \\
$\Delta \mathrm{PM}_{\mathrm{t}}$ & -0.2004 & 0.3326 & 0.1265 & 0.2563 & 1.0000 & & & & \\
$\mathrm{ATO}_{\mathrm{t}}$ & -0.1214 & 0.2649 & 0.1667 & 0.1709 & 0.0778 & 1.0000 & & & \\
$\mathrm{PM}_{\mathrm{t}}$ & -0.3341 & 0.8365 & 0.1096 & & 0.3452 & -0.1375 & 1.0000 & & \\
$\Delta \mathrm{ATO}_{\mathrm{t}+1}$ & 0.3519 & -0.1400 & -0.2758 & 0.1811 & & & -0.123 & 1.0000 & \\
$\Delta \mathrm{PM}_{\mathrm{t}+1}$ & 0.7430 & -0.3069 & -0.2218 & & -0.0182 & & -0.283 & 0.2622 & 1.0000 \\
\hline
\end{tabular}

(*) Solo se muestran los coeficientes significativos al nivel del 10\%. Fuente: Elaboración propia

En la Figura 2 el gráfico de dispersión (scatterplots) muestra la relación entre los residuos y la variable pronosticada, este gráfico proporciona una prueba para los supuestos de normalidad, linealidad y homocedasticidad (Tabachnick y Fidell, 2001). Cuando se cumplen estos supuestos, la trama de los residuos debería parecerse a una banda rectangular con la concentración de los residuos a lo largo del centro. En el caso de inexistencia de linealidad, la forma general de la gráfica de dispersión es curva en lugar de rectangular y si la banda es más ancha a mayores valores pronosticados, o en forma de embudo, indica la presencia de heterocedasticidad (Tabachnick y Fidell, 2001). Por otra parte, la tabla 4 presenta las pruebas estadísticas de los supuestos básicos del MCO. El incumplimiento de algunos de los supuestos afecta las inferencias estadísticas, conduciendo a conclusiones incorrectas.

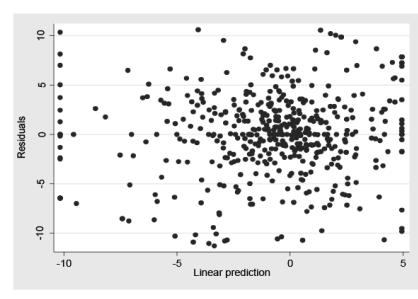

(l)

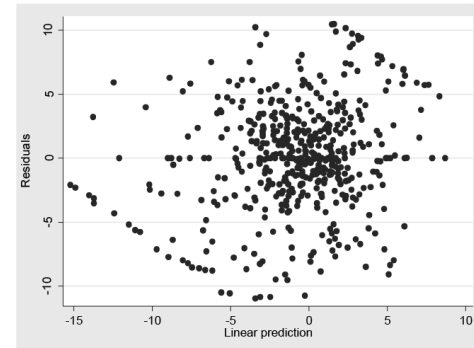

(IV)

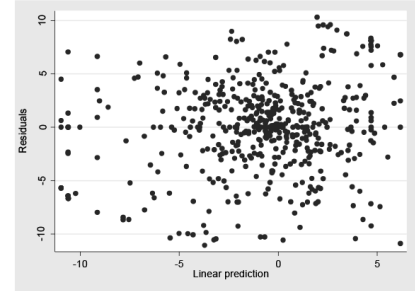

(II)

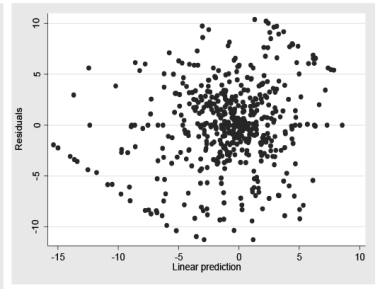

(III)

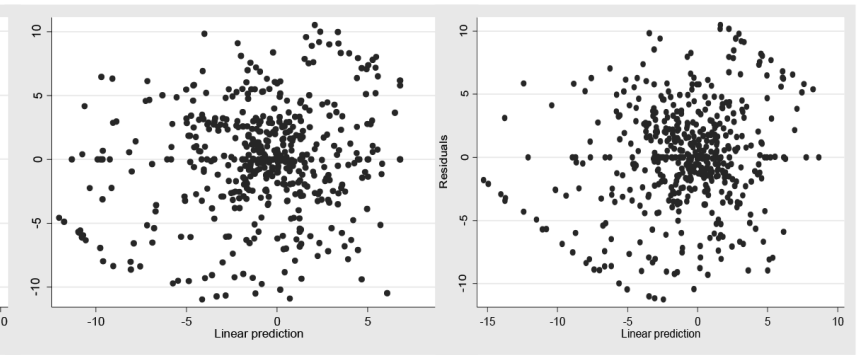

(V) 


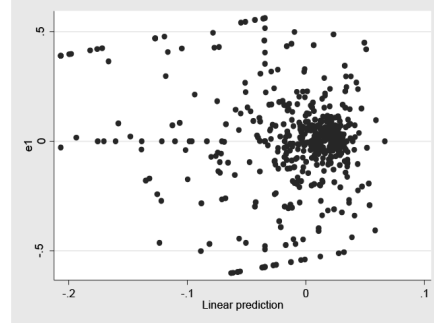

(a)

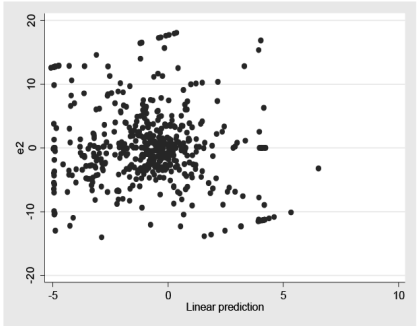

(b)

Figura 2. Scatterplot de los errores con los valores estimados de la variable dependiente para cada uno de los modelos (indicado entre paréntesis).

Fuente: Elaboración propia.

Tabla 4

Pruebas de especificación de los modelos

\begin{tabular}{cccccccccc} 
& \multicolumn{8}{c}{ Modelos } \\
\hline & I & II & III & IV & V & VI & a & b \\
\hline $\begin{array}{c}\text { Test de normalidad } \\
\text { chi2(2) }\end{array}$ & $\mathbf{2 . 2 8}$ & $\mathbf{4 . 2 8}$ & $\mathbf{2 . 6 2}$ & $\mathbf{1 . 6 3}$ & $\mathbf{3 . 8 7}$ & $\mathbf{2 . 2 7}$ & 15.28 & $\mathbf{3 . 1 2}$ \\
\hline P-valor & 0.3197 & 0.1177 & 0.2701 & 0.4427 & 0.1441 & 0.3219 & 0.005 & 0.2097
\end{tabular}

Test de

heteroscedasticidad

\begin{tabular}{ccccccccc}
\hline $\begin{array}{c}\text { Breusch-Pagan / Cook- } \\
\text { Weisberg }\end{array}$ & 1.390 & 0.090 & 0.020 & 0.010 & 0.020 & 0.040 & $\mathbf{3 3 . 0 7}$ & 2.310 \\
\hline P-valor & 0.239 & 0.767 & 0.893 & 0.925 & 0.879 & 0.842 & 0.000 & 0.129 \\
\hline
\end{tabular}

Test de correlación serial

\begin{tabular}{|c|c|c|c|c|c|c|c|c|}
\hline Durbin-Watson & 1.851 & 1.837 & 1.813 & 1.866 & 1.794 & 1.843 & 1.828 & 1.874 \\
\hline $\begin{array}{c}\text { Valor crítico (DL) } \\
\square=0.05\end{array}$ & 1.846 & 1.831 & 1.831 & 1.818 & 1.827 & 1.818 & 1.840 & 1.840 \\
\hline $\begin{array}{c}\text { Valor crítico (DU)- } \\
\square=0.05\end{array}$ & 1.854 & 1.866 & 1.866 & 1.879 & 1.870 & 1.879 & 1.857 & 1.857 \\
\hline \multicolumn{9}{|l|}{$\begin{array}{c}\text { Test de } \\
\text { multicolinealidad -VIF }\end{array}$} \\
\hline $\mathrm{RNOA}_{\mathrm{t}}$ & 1 & 1.06 & 1.17 & 1.19 & 1.16 & 1.19 & & \\
\hline $\mathrm{CrNOA}_{t}$ & & & 1.18 & 1.18 & & 2.07 & & \\
\hline$\Delta \mathrm{ATO}_{\mathrm{t}}$ & & & 1.13 & 1.91 & & 1.93 & & \\
\hline$\Delta \mathrm{PM}_{\mathrm{t}}$ & & & 1.15 & 2.00 & 1.12 & 1.14 & & \\
\hline$\Delta$ PM y $\nabla$ ATO & & 1.34 & & 1.8 & & & & \\
\hline$\nabla$ PM у $\Delta$ ATO & & 1.37 & & 1.94 & & & & \\
\hline$\nabla$ PM у $\nabla$ ATO & & 1.44 & & 3.05 & & & & \\
\hline$\Delta$ ATO y $\nabla$ CrNOA & & & & & 1.59 & 2.29 & & \\
\hline$\nabla$ ATO y $\Delta$ CrNOA & & & & & 1.60 & 2.35 & & \\
\hline$\nabla$ ATO y $\nabla$ CrNOA & & & & & 1.51 & 2.48 & & \\
\hline ATO & & & & & & & 1.00 & \\
\hline$\Delta \mathrm{ATO}_{\mathrm{t}}$ & & & & & & & 1.00 & \\
\hline $\mathrm{PM}$ & & & & & & & & 1.17 \\
\hline$\Delta \mathrm{PM}_{\mathrm{t}}$ & & & & & & & & 1.17 \\
\hline
\end{tabular}

Fuente: Elaboración propia 
Los gráficos de la figura 2 y las pruebas de especificación de la tabla 4 fueron obtenidos para los distintos modelos planteados por el MCO clásico. El test de asimetría y curtosis indica una distribución normal de los errores con excepción del modelo "a", no obstante, el gráfico de dispersión correspondiente muestra una distribución aproximada a la normal. El test de heteroscedasticidad muestra que no se cumple el supuesto de igualdad de varianzas en el caso del modelo "a". En caso del supuesto de no autocorrelación, el test de Durbin-Watson indica que, si bien la mayor parte de los modelos se ubican en la zona de indefinición, con excepción de los modelos III, V y "a" en la zona de rechazo y el modelo "b" en la zona de aceptación de la hipótesis nula. El factor de inflación de la varianza (FIV) es muy inferior a 10, lo cual indica la inexistencia de colinealidad.

En los estudios de finanzas, habitualmente, son utilizados datos de panel, estos contienen observaciones de múltiples empresas y múltiples períodos de tiempo, la estimación por el MCO puede producir residuos correlacionados a través de las empresas y el tiempo. La violación de los supuestos de homocedasticidad y no correlación serial por MCO produce errores estándar de los estimadores sesgadas que son cruciales en el testeo de las hipótesis. El problema es resuelto por el método de errores estándar clustered (agrupados) por empresa y año (Thompson 2011; Cameron, Gelbach, y Miller 2009). En este método la matriz de la varianza de los estimadores por MCO se obtiene de la suma de estimación de las varianzas estimadas que se agrupan por empresa y tiempo, menos la habitual matriz de varianzas por MCO robusta a la heterocedasticidad (Thompson 2011). Peterson (2009) sostiene que los errores estándar clustered por empresa y año resultan más adecuados para la corrección de los residuos que otros de los métodos empleados (White, Newey-West, Fama-MacBeth y modelos de efectos fijos y aleatorios). De acuerdo con el método, los coeficientes de los estimadores no resultan modificados, si la matriz de varianzas-covarianzas.

El estadístico de test de Voung es utilizado para contrastar la significatividad entre los $R^{2}$ de dos modelos que tienen las mismas variables dependientes pero distintas variables independientes. Un valor positivo (negativo) indica un mejor (peor) poder explicativo de cada uno de los modelos en relación con el modelo I, utilizado como referencia.

\section{Discusión de los resultados}

En la tabla 5 se muestran los resultados obtenidos de los distintos modelos para la predicción del cambio de la RNOA del año siguiente. En el modelo I, el coeficiente de RNOA es significativo e indica una relación inversa con el $\Delta \mathrm{RNOA}_{\mathrm{t}+1}$, por cual a un mayor valor de la rentabilidad actual tenemos una menor rentabilidad futura y viceversa. El coeficiente obtenido $(\beta=-0.390 ; t=-13.43)$ es muy superior a los resultados obtenidos para Estados Unidos por Penman y Zhang (2002) $\left(\beta_{1}=-0.176\right)$ y Farfield y Yohn (2001) $\left(\beta_{1}=-0.1492\right)$, indicando un proceso de reversión a la media más pronunciado, lo cual podría obedecer al contexto de inestabilidad. 
Tabla 5

Resultados de la regresión por cada modelo

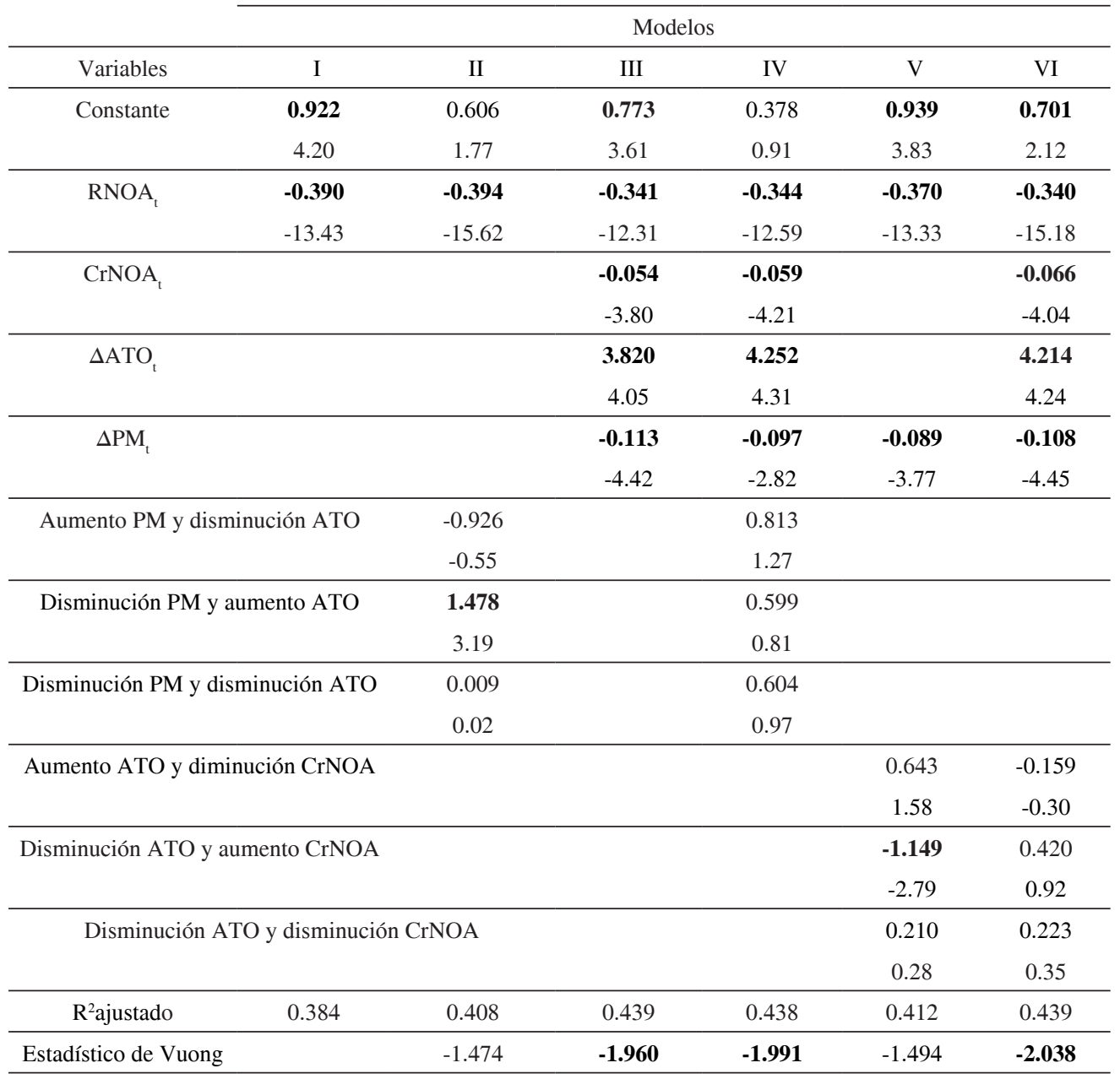

(*) Debajo de cada coeficiente es indicado el estadístico $t$, los coeficientes en negrita indica que son significativos al nivel del 10\%. Fuente: Elaboración propia.

En el modelo II no muestra un mayor pode explicativo que el modelo I, el estadístico de Vuong no resulta significativo. El coeficiente de la RNOA actual es significativo y de un valor muy similar al modelo I. El coeficiente de $\mathrm{RM} 2\left(\Delta \mathrm{ATO}_{\mathrm{t}}>0\right.$ y $\left.\Delta \mathrm{PM}_{\mathrm{t}}<0\right)$ significativo y positivo $\left(\beta_{3}=1.478 ; \mathrm{t}=3.19\right)$, indica que tiene un mayor efecto $\left(\Delta \mathrm{ATO}_{\mathrm{t}}>0\right.$ y $\left.\Delta \mathrm{PM}_{\mathrm{t}}>0\right)$, representado por la ordenada de origen. El coeficiente de RM2 positivo y significativo podría ser atribuido al efecto de earnings management (manipulación de resultados), si no fuese porque el efecto opuesto representado por $\operatorname{RM} 1\left(\Delta \mathrm{ATO}_{\mathrm{t}}<0\right.$ y $\left.\Delta \mathrm{PM}_{\mathrm{t}}>0\right)$ no es significativo. De lo cual surge que el aumento de la $\mathrm{ATO}_{\mathrm{t}}$ y $\mathrm{PM}_{\mathrm{t}}$ no tiene un efecto perdurable en el tiempo, posiblemente, por la necesidad de ajustar los gastos en el tiempo ante un mayor nivel de ventas. 
En el modelo III, exhibe un mayor poder explicativo que el modelo I ( $\mathrm{R}^{2}$ ajustado: de 0.4390 a 0.384 ) que resulta significativo de acuerdo con el estadístico de Vuong, por el efecto el efecto adicional de la desagregación de Dupont. Los coeficientes son todos significativos, los coeficientes del $\operatorname{CrNOA}_{\mathrm{t}}\left(\beta_{2}=-0.054 ; \mathrm{t}=-3.80\right)$ y del $\Delta \mathrm{PM}_{\mathrm{t}}\left(\beta_{4}=-0.113 ; \mathrm{t}=-4.42\right)$ son de signo negativo y del $\Delta \mathrm{ATO}_{\mathrm{t}}\left(\beta_{3}=3.820 ; \mathrm{t}=4.05\right)$ es de signo positivo, con respecto $\Delta \mathrm{RNOA}_{\mathrm{t}+1}$. En síntesis, la única variable que predice un aumento sostenible de la rentabilidad futura es el incremento del $\Delta \mathrm{ATO}_{\mathrm{t}}$, lo cual es consistente con diferentes estudios; mientras que, el $\Delta \mathrm{PM}_{\mathrm{t}}$ tiene un efecto negativo, de acuerdo con el estudio de Bauman (2014), no así con Farfield y Yohn (2001). En el modelo IV, los coeficientes que capturan la interacción entre los signos del cambio del margen y rotación coeficientes no son significativos, por lo tanto, este modelo no brinda información relevante con relación al modelo I, de acuerdo con el estadístico de Vuong.

En el modelo V, exhibe un mayor poder explicativo que el modelo I, pero acuerdo al estadístico de Vuong no resulta significativo. Los resultados muestran un efecto negativo de los coeficientes de $\operatorname{RNOA}_{t}\left(\beta_{1}=-0.370 ; \mathrm{t}=-13.33\right)$ y $\Delta \mathrm{PM}_{\mathrm{t}},\left(\beta_{2}=-0.089 ; \mathrm{t}=-3.77\right)$. La variable GR1 que representa a $\left(\Delta \mathrm{ATO}_{\mathrm{t}}<0\right.$ y $\left.\mathrm{CrNOA}_{\mathrm{t}}>0\right)$ es negativa y significativa $\left(\beta_{4}=-1.149\right.$; $\mathrm{t}=-2.79)$ en relación con la ordenada de origen positiva y significativa $(\mathrm{D}=0,939 ; \mathrm{t}=3.83)$ que refleja $\left(\Delta \mathrm{ATO}_{\mathrm{t}}>0\right.$ y $\left.\mathrm{CrNOA}_{\mathrm{t}}>0\right)$. Esto indica que el mayor efecto corresponde a $\left(\Delta \mathrm{ATO}_{\mathrm{t}}>0\right.$ y $\mathrm{CrNOA}_{\mathrm{t}}>0$ ) y es el caso donde las nuevas inversiones producen un aumento de las ventas. Los coeficientes de las variables GR2 y GR3, si bien son positivos no son significativos.

Finalmente, el modelo VI agrega al modelo III la relación entre el $\Delta \mathrm{ATO}_{\mathrm{t}}$ y $\mathrm{CrNOA}_{\mathrm{t}}$. Las variables agregadas no tienen un efecto significativo sobre el $\Delta \mathrm{RNOA}_{t+1}$, por lo tanto, los resultados son similares al modelo III, en cuanto al poder explicativo y significatividad de los coeficientes.

Tabla 6

Predicción del margen y rotación

\begin{tabular}{ccc}
\hline Variable dependiente & $\Delta \mathrm{ATO}_{\mathrm{t}+1}$ & $\Delta \mathrm{PM}_{\mathrm{t}+1}$ \\
\hline Constante & $\mathbf{0 . 0 4 7}$ & $\mathbf{0 . 8 6 1}$ \\
& 4.10 & 3.62 \\
\hline $\mathrm{ATO}_{\mathrm{t}}$ & $\mathbf{- 0 . 0 5 7}$ & \\
& -3.38 & \\
\hline$\Delta \mathrm{ATO}_{\mathrm{t}}$ & $\mathbf{0 . 2 9 8}$ & \\
\hline $\mathrm{PM}_{\mathrm{t}}$ & 4.52 & $\mathbf{- 0 . 1 5 7}$ \\
& & -4.24 \\
$\Delta \mathrm{PM}_{\mathrm{t}}$ & & $\mathbf{- 0 . 2 9 5}$ \\
& & -6.79 \\
\hline $\mathrm{R}^{2}$ ajustado & 0.186 & 0.311 \\
\hline
\end{tabular}

(*) Para cada coeficiente es indicado el estadístico t, los coeficientes en negrita indica que son significativos al nivel del $10 \%$. Fuente: Elaboración propia. 
En la tabla 6 se informan los resultados obtenidos de los modelos para la predicción del cambio del PM y ATO del año siguiente. Una primera cuestión, en ambos modelos los coeficientes de $\mathrm{ATO}_{\mathrm{t}}\left(\beta_{1}=-0.057 ; \mathrm{t}=-3.38\right)$ y $\mathrm{PM}_{\mathrm{t}}\left(\beta_{1}=-0.157 ;=\mathrm{t}-4.24\right)$ son significativos y negativos, por lo cual el proceso de reversión de la RNOA es producido tanto por la reversión de $\mathrm{ATO}_{\mathrm{t}}$ y $\mathrm{PM}_{\mathrm{t}}$. Por otro lado, el $\Delta \mathrm{ATO}_{\mathrm{t}}$ exhibe un efecto positivo y significativo $\left(\beta_{2}=0.298\right.$; $\mathrm{t}=4.52)$ sobre el $\Delta \mathrm{ATO}_{\mathrm{t}+1}$ y el $\Delta \mathrm{PM}_{\mathrm{t}}$ exhibe un efecto negativo y significativo $\left(\beta_{3}=-0.295\right.$; $\mathrm{t}=-6.79$ ) sobre y $\Delta \mathrm{PM}_{\mathrm{t}+1}$. Lo cual confirma el efecto positivo del cambio de la rotación $\mathrm{y}$ negativo del cambio del margen de ganancias sobre la rentabilidad del año siguiente.

\section{Conclusiones}

El presente trabajo tiene como objetivo evaluar la capacidad predictiva en el corto plazo, de la rentabilidad de los activos operativos netos actual y del cambio en el margen de ganancias y rotación de los activos operativos netos actuales. El cálculo de la rentabilidad es calculado por los componentes permanentes de los resultados y con datos ajustados por inflación. De la discusión de los diferentes modelos planteados para predicción del cambio de la RNOA del año siguiente, surgen las siguientes conclusiones:

a) En el análisis previo, a nivel agregado, la tendencia de la media anual de la rotación muestra un comportamiento más estable de la rotación que el margen, siendo afectado principalmente por el nivel de actividad.

b) El cambio en el margen de ganancias y rotación de activos muestra un poder predictivo adicional a la rentabilidad actual.

c) La RNOA del año siguiente exhibe fuerte reversión con respecto RNOA actual, inclusive es superior a estudios similares en países desarrollados. Lo cual es producido por la reversión de la rotación de los activos y del margen de ganancias.

d) El crecimiento de los activos operativos netos muestra un efecto negativo sobre la RNOA del año siguiente, probablemente, sea ocasionado por los criterios conservadores de contabilización.

e) El efecto de earnings management, controlado a través de la relación ATO-PM, mostró no tener un efecto relevante, lo cual es bastante lógico, considerando que las partidas que componen los resultados analizadas son las que hacen a la actividad operativa principal.

f) El efecto conjunto de una disminución en el margen ganancias y aumento de la rotación tiene un efecto superior sobre la RNOA del año siguiente que el aumento de ambos. Esto resulta consistente con el hecho que el cambio del margen de ganancias no es persistente en el tiempo.

g) El cambio en la rotación de los activos exhibe un efecto positivo sobre el cambio de la RNOA del año siguiente, la eficiencia permite la mejoría en la rentabilidad. A su vez, individualmente, el cambio en la rotación muestra un efecto positivo sobre el cambio de la rotación del año siguiente.

h) El aumento del margen de ganancias revierte rápidamente, determinando un efecto negativo sobre la RNOA del año siguiente, además, muestra tener un efecto negativo sobre el cambio del margen de ganancias del año siguiente. 


\section{Referencias}

Aguiar, M. y Gopinath, G. (2007). Emerging market business cycles: The cycle is the trend. Journal of Political Economy 115: 69-102.http://dx.doi.org/10.1086/511283

Amir, E., Kama, I. y Levi, S. (2015). Conditional Persistence of Earnings Components and Accounting Anomalies. Journal of Business Finance \& Accounting, 42, 801-825. http://dx.doi:10.1111/jbfa.12127

Anuarios del Instituto Argentino del Mercado de Capitales-IAMC (2002-2012). Disponible en http: //www.iamc.sba. com.ar/informes/informe_anuario/. Consultado: 30/04/2014.

Arbanell, J.S. y Bushee B. J. (1997). Fundamental analysis, future earnings, and stock prices. Journal of Accounting Research, 35, 1-24. http://dx.doi.org/ 10.2307/249 1464

Bauman, M. P. (2014). Forecasting operating profitability with DuPont analysis. Review of Accounting and Finance, 13(2), 191-205. http://dx.doi:10.1108/raf-11-2012-0115.

Canarella, G.; Miller, S.M. y Nourayi, M.M. (2013). Firm profitability: Mean-reverting or random-walk behavior? Journal of Economics and Business, 66, 76- 97. http://dx.doi.org/10.1016/j.jeconbus.2012.11.002

Cameron, A. C., Gelbach, J., y Miller, D. L. (2009). Robust Inference with Multiway Clustering. NBER Technical Working Paper Number 327. https://doi.org/ 10.3386/t0327

Esplin, A., Hewitt, M., Plumlee, M. y Yohn, T. L. (2013). Disaggregating operating and financing activities. Implications for forecasts of future profitability. Review of Accounting Studies, 19, 328-362. https://doi.org/10.1007/ s11142-013-9256-5

Fama, E. F. y French K.R. (2000). Forecasting Profitability and Earnings. The Journal of Business, 73(2), 161-175. https://doi.org/10.1086/209638

Fairfield, P., R. Sweeneyy T. Yohn, T.L. (1996). Accounting Classification and the predictive Content of Earnings. The Accounting Review, 71, 337-355. Obtenido de https://www.jstor.org/stable/248292. Consultado: 01/07/2015.

Farfield, P.M. y Yohn T.L. (2001). Using asset turnover and profit margin to forecast changes in profitability. Review of Accounting Studies, 6, 371-385. https://doi.org/10.1023/a:1012430513430

Fondo Monetario Internacional (2013). Perspectivas Políticas Mundiales-octubre 2013. Disponible en http: //www. imf.org/external/spanish/pubs/ft/weo/2013/02/pdf/texts. pdf, Consultado: 18/10/2013.

Hejazi, R., Adampira S., Bahrami Z. M. (2016). A Diagnostic for Earning Management by Using Changes in Asset Turnover and Profit Margin. The Financial Accounting and Auditing Researches, 8 (29), 73- 95. Obtenido de http://www.sid.ir/En/ Journal/ViewPaper.aspx?ID=483292. Consultado: 01/07/2016.

Jansen, I., Ramnath, S. y Yohn, T.L. (2011). A diagnostic for earnings management using changes in asset turnover and profit margin. Contemporary Accounting Research, 29 (1), 221-251. https://doi.org/10.1111/j.19113846.2011.01093.x

Konchitchki, Y. (2011). Inflation and Nominal Financial Reporting: Implications for Performance and Stock Prices. The Accounting Review 86(3), 1045-1085. http://dx.doi.org/10.2308/accr.00000044

Lev, B. y Thiagarajan S. (1993). Fundamental information analysis. Journal of Accounting Research31: 190-215. http://dx.doi.org/10.2307/2491270

Lipe, R. y Kormendi, R. (1994). Mean Reversion in Annual Earnings and Its Implications for Security Valuation. Review of Quantitative Finance and Accounting 4: 24-46. http://dx.doi.org/10.1007/BF01082663

Mahmud, R., Ibrahim, M. K., y Pok, W. C. (2009). Earnings Quality Attributes and Performance of Malaysian Public Listed Firms. http://dx.doi.org/10.2139/ssrn.1460309

Missim D. y Penman, S.H. (2001). Ratio Analysis and Equity Valuation: From research to practice. Review of Accounting Studies, 6,109-154. https://doi.org/ 10.1023/A:1011338221623

Monterrey, J. y Sánchez-Segura, A. (2011). Persistencia y capacidad predictiva de márgenes y rotaciones: un análisis empírico. Revista de Contabilidad-Spanish Accounting Review, 14(1), 121-153. https://doi.org/10.1016/S11384891(11) 70024-3

Monterrey Mayoral, J y Sánchez Segura, A (2017). Una evaluación empírica de los métodos de predicción de la rentabilidad y su relación con las características corporativas. Revista de Contabilidad-Spanish Accounting Review, 20(1), 95-106. http://dx.doi.org/10.1016/j.rcsar.2016.08.001. 
Mueller, D. C. (1977). The Persistence of Profits above the Norm. Economica, 44(176), 369-380. https://doi. org/10.2307/2553570

Notz, S. y Rosenkranz, P. (2014). Business cycles in emerging markets: the role of liability dollarization and valuation effects. Working paper $\mathrm{N}^{\circ} 163$ University of Zurich. https://doi.org/10.2139/ssrn.2459001

Ohlson, J. (1995). Earnings, book values, and dividends in equity valuation. Contemporary Accounting Research, 22(2), 661-687. https://doi.org/ 10.1111/j.1911-3846. 1995.tb00461.x

Ou, J.A. (1990). The information content of nonearnings accounting numbers as earnings predictors. Journal of Accounting Research, 28, 144-163. http://dx.doi.org/ 10.2307/2491220

Penman, S.H. y Zhang X. (2002). Modeling sustainable earnings and P/E ratios with financial statement analysis. Working paper de la Columbia University y University of California, Berkeley. http://dx.doi.org/10.2139/ssrn.318967

Petersen, M. A. (2009). Estimating standard errors in finance panel data sets: Comparing approaches. Review of financial studies, 22(1), 435-480. http://dx.doi.org/ 10.1093/rfs/hhn053

Richardson, S., Tuna, I. y Wysocki, P (2010). Accounting anomalies and fundamental analysis: A review of recent research advances. Journal of accounting \& economics, 50(2-3), 410-454. http://dx.doi.org/10.1016/j.jacceco.2010.09.008

Sandin, A. y Porporato, M. (2007). Corporate bankruptcy prediction models applied to emerging economies. Evidence from Argentina in the years 1991-1998. International Journal of Commerce and Management, 17(4), $295-311$. http://dx.doi.org/10.1108/1056921071084437.

Selling, T.I. y Stickney, C. (1989). The effects of Business Environment and Strategy on a Firm's Rate of Return on Assets. Financial Analysts Journal, 45(1), 43-68. https://doi.org/10.2469/faj.v45.n1.43

Soliman, M. T. (2004). Using Industry-Ajusted DuPont Analysis to Predict Future Profitability. Working Paper Working Paper Stanford University. http://dx. doi.org/10. 2139/ssrn.456700.

Stigler, G. (1963). Capital and Rates of Return in Manufacturing Industries. Princeton, NJ: Princeton University Press.

Swanson, E. P., Rees, L. y Juárez-Valdés, L. F. (2003). The contribution of fundamental analysis after a currency devaluation. The Accounting Review, 78(3), 875-902. https://doi.org/10.2308/accr.2003.78.3.875

Tabachnick, B.G y Fidell, L.S. (2001). Using Multivariate Statistics (4th Ed). New York: Allyn \& Bacon.

The Economist (2013). When giants slow down. Disponible en http://www.economist. com/ news/briefing/21582257-most-dramatic-and-disruptive-period-emerging-mar ket-growth- world-has-ever-seen. Consultado: 20/11/2014

Thompson, S. B. (2011). Simple formulas for standard errors that cluster by both firm and time. Journal of financial Economics, 99(1), 1-10. http://dx.doi.org/ 10.1016/j.jfineco.2010.08.016

Zanjirdar, M., Khaleghi Kasbi P. y Madahi Z. (2014). Investigating the effect of adjusted DuPont ratio and its components on investor's decisions in short and long term- Management Science Letters, 4,591-596. http://dx.doi. org/10.5267/j.msl. 2014.1.003 